\title{
Patrones en el conocimiento, uso y manejo de plantas en pueblos indígenas de México
}

\author{
Javier Caballero*, Alejandro Casas**, Laura Cortés* y Cristina Mapes*
}

\section{RESUMEN}

Se presenta una síntesis de información etnobiológica obtenida entre grupos indígenas de México, analizando tendencias y patrones en las formas de conocer, utilizar y manipular los recursos biológicos. Los sistemas indígenas de nomenclatura y clasificación de plantas son altamente elaborados y complejos. Aunque en general éstos parecen seguir los principios generales de clasificación folk descubiertos por Brent Berlin y otros autores, se observan algunas categorías taxonómicas y sistemas de nomenclatura estrechamente asociados a las formas de uso de los recursos locales. La subsistencia de las poblaciones indígenas ha estado basada en una estrategia que combina diferentes prácticas productivas y que aprovecha una gran diversidad de productos disponibles tanto espacial como temporalmente. Esta estrategia involucra también el aprovechamiento integral de un amplio espectro de especies de plantas y variabilidad genética intraespecífica bajo diferentes niveles de domesticación.

\section{ABSTRACT}

An overview of ethnobiological information obtained among Indian peoples of Mexico is

Jardín Botánico, Instituto de Biología, Universidad Nacional Autónoma de México.

Apartado Postal 70-614, D.F 04510, México. jcnieto@servidor.unam.mx

Instituto de Ecología, Campus Morelia, Universidad Nacional Autónoma de México.

Apartado Postal 27-3 (Xangari), Morelia, Michoacán 58089, México. presented, analyzing patterns and tendencies in the forms of perceiving, using, and manipulating plant resources. The plant classification folk systems studied are highly complex. Although in general they follow the general principles formulated by Brent Berlin and other scholars, some significant variations are observed. Subsistence has been based on a strategy which combines different productive activities and takes advantage of a wide variety of plant products available both in time and space. This subsistence strategy also involves managing a wide variety of resources under different degrees of domestication by humans.

\section{Introducción}

El territorio mexicano es una región cultural y ecológicamente diversa. Su posición geográfica dentro del área de confluencia de los reinos biogeográficos Neártico y Neotropical, su compleja topografía, así como las diferencias ambientales debidas a la altitud y a la latitud, hacen que esta región sea un complejo mosaico ecológico. Debido a lo anterior, la cubierta vegetal de esta región es una de las más variadas de la tierra, pues incluye desde los desiertos hasta las selvas tropicales, los bosques templados y los páramos de altura. Esto explica la enorme diversidad vegetal que existe a nivel de especies. Se calcula que en México existen entre 25 y 30,000 especies de plantas vasculares (Rzedowski 1978, 1993; Toledo 1988). Por otra parte, esta región posee una gran riqueza cultural debido al gran número de grupos étnicos que la habitan y al alto grado de desarrollo civilizatorio alcanzado en la antigüedad. En México existen actualmente todavía más de 50 grupos étnicos y es conocido que durante la época prehispánica en la parte meridional del terrritorio 
mexicano se desarrollaron algunas de las más grandes civilizaciones de la antigüedad. La riqueza biológica de México, su diversidad cultural, así como su larga historia de poblamiento, se han traducido en el desarrollo de una vasta tradición etnobotánica, la cual incluye el conocimiento, el uso y el manejo de una gran cantidad de especies vegetales a través de complejas formas de interacción entre las comunidades locales y su entorno vegetal. El análisis comparativo preliminar de los datos etnobotánicos recopilados por los autores de este trabajo entre diversos grupos étnicos de México, así como los existentes en la vasta literatura sobre el tema, indica que a pesar de la diversidad ecológica y cultural de la región existen tendencias comunes en la percepción, la clasificación, la utilización y el manejo de los recursos vegetales entre la población indígena. En el presente trabajo se describen en forma general estos patrones y se plantea la importancia teórica que tiene en Etnobiología.

\section{Patrones de Percepción y Clasificación de las Plantas}

El estudio de las formas de percepción y clasificación de las plantas es un área de la Etnobiología derivada directamente de la Linguística, particularmente de la corriente conocida como Etnografía Semántica o Antropología Cognoscitiva. Hacia finales de la década de los años '60, Charles Frake (1962) planteó que en Lingüística lo importante no es recopilar los nombres de las cosas sino más bien entender los principios que subyacen en las maneras como se nombran a las cosas. Bajo esta perspectiva se desarrolló una corriente antropológica conocida como Etnografía Semántica, para la cual la cultura, o una dimensión de ella, constituye un fenómeno racional y puede ser entendida como conocimiento. Es en este contexto que las formas de percepción, clasificación y organización de las discontinuidades del mundo natural pueden considerarse como indicadores de la cultura; y su estudio comparativo permite descubrir fenómenos culturales universales, es decir, rasgos comunes a todo el género humano, independientes de los contextos históricos y geográficos específicos. Fue bajo estos principios que a partir de los años setenta se realizaron numerosos estudios que documentaron las formas de nombrar a las plantas y los animales por diferentes grupos indígenas en varias partes del mundo. El trabajo realizado por Brent Berlín y sus colaboradores (Berlin et al. 1973 y 1974) entre los Mayas Tzeltales de los altos de Chiapas, en México, fue la piedra de toque para el desarrollo de los estudios de los sistemas folk o tradicionales de clasificación biológica, los cuales se convirtieron en un nuevo campo de interés para la Etnobiología. Estos autores recopilaron y analizaron los nombres para las plantas conocidas por los Tzeltales de esta región de México y encontraron que esta etnia tiene un complejo sistema de nomenclatura, el cual es a su vez la expresión de un sofisticado sistema de clasificación botánica. Cuando este sistema se comparó con los encontrados en otras partes del mundo se pudo ver que existía una serie de principios universales en la manera de clasificar y de nombrar las plantas. De esta manera Berlin y sus colaboradores concluyeron que todo grupo humano sigue siempre un mismo sistema para nombrar las plantas y los animales de su entorno (Berlin et al. 1973). Este es un sistema jerárquico, el cual incluye seis niveles de inclusividad (fig. 1). El primer nivel es el más inclusivo y es el que contiene a todas las plantas o los animales según sea el caso. Este principio único se divide generalmente en unas pocas formas de vida, normalmente árboles, arbustos y enredaderas, las que a su vez incluyen a los taxa genéricos los cuales representan la mayor parte de los taxa que se pueden reconocer en estos sistemas. Es común también la existencia de taxa no bien definidos, al nivel forma de vida entre los que se incluyen formas de vida "atípicas" o "aberrantes", tales como los de palmas, agaváceas y cactáceas. Aquellos géneros de plantas que tienen una significación cultural pueden ser subdivididos en varios taxa más. Estos taxa fueron llamados taxa específicos por Berlin y colaboradores (1973). Estos autores observaron también que aquellas especies de mayor importancia económica son a su vez agrupadas en unos pocos taxa a nivel varietal. Los aportes de Berlin y sus colaboradores fueron de gran significado para la Antropología porque mostraron evidencia sobre la existencia de aspectos universales en la cultura. Los estudios sobre las taxonomías folk revelaron que en realidad existen elementos comunes, en la manera de conocer, nombrar y clasificar las discontinuidades del mundo natural. Esta evidencia apoya la idea de la existencia de aspectos universales en la cultura y mues- 


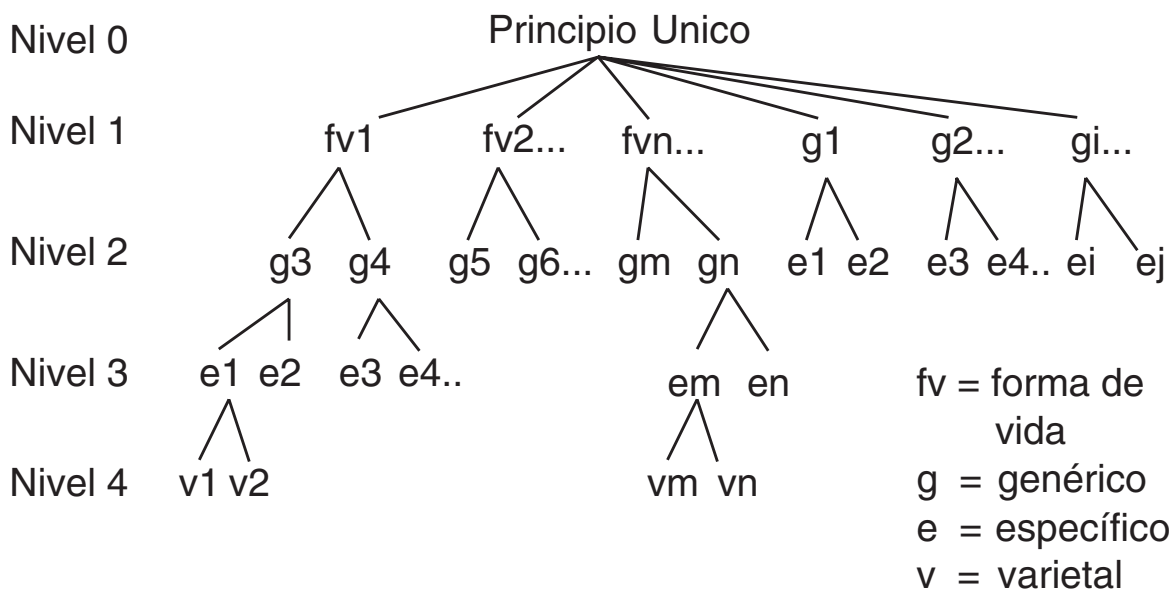

Figura 1. Estructura de las clasificaciones folk de acuerdo a Berlin y colaboradores (1973).

tra además que estos sistemas de clasificación resultan ser muy precisos, muy detallados y que son completamente comparables a los de la taxonomía Lineana.

La mayor parte de los estudios sobre los sistemas de clasificación biológica realizados entre otros grupos étnicos de México (Barrera et al. 1976; Hunn 1977; Toledo y cols. 1981; Mapes et al. 1981; 1982; Argueta 1988; Williams 1990), coinciden en general con lo descrito por Berlin y sus colaboradores para los Maya Tzeltales. De este modo Barrera (1979) y Barrera y cols. (1976) reportan que en Maya Yucateco las plantas se dividen en tres grandes grupos: che' (árboles y arbustos, o plantas leñosas en general); xiw (plantas herbáceas) y $A k^{\prime}$ o Kan (plantas trepadoras y rastreras). En el nivel forma de vida existen también grupos de plantas pertenecientes a familias botánicas con formas biológicas distintivas o "aberrantes", tales como las palmas, los agaves o los cactos. El resto de la clasificación sigue en forma cercana los principios descritos por Berlin y colaboradores (fig. 2). Los estudios de otras clasificaciones tradicionales en Mesoamérica han mostrado resultados contrastantes. De este modo, en el caso de la clasificación micológica Purhepecha se observa que aunque se nombra el principio único terekuicha (los hongos), los taxa de nivel forma de vida ni son nombrados ni son claramente reconocibles (Mapes et al. 1981, 1982). En contraste, en esta clasificación los taxa genéricos son muy numerosos y claramente definidos. El examen de la clasificación botánica Purhepecha muestra algunas variantes importantes no previstas en el modelo de Berlin y sus colaboradores, particularmente en lo que se refiere a los taxa forma de vida. Si bien existen los taxa correspondientes a árbol, hierba o bejuco, en esta clasificación existe un taxon del nivel forma de vida, el cual agrupa a las plantas con flores vistosas o aparentes. Asimismo tanto en Nahua como en Purhepecha, en Mixteco y algunas otras clasificaciones mesoamericanas, puede reconocerse la existencia de un taxon de nivel forma de vida el cual agrupa a todas las plantas, cuyas flores, hojas o tallos tiernos pueden comerse herventados o guisados a manera de verdura (Toledo et al. 1980; Caballero y Mapes 1985; Casas, Viveros y Caballero 1994; Casas y Caballero 1996). Estas plantas son nombradas quilitl en Nahuatl, xakua en Purhepecha y yiwa en Mixteco. Un hecho frecuentemente observado en las clasificaciones biológicas mesoamericanas es la existencia de taxa de la misma jerarquía pero que están asociados a uno que es considerado como el verdadero o el más importante, de modo que todos los taxa de ese grupo son referidos o nombrados en relación con el taxon principal. Por ejemplo, en la clasificación micológica purhepecha el taxon kux tereko incluye a las especies Hypomyces lactifluorum, Gomphus floccossus e Hygrophoropsis aurantiaca (fig. 3), pero es sólo la primera espe- 


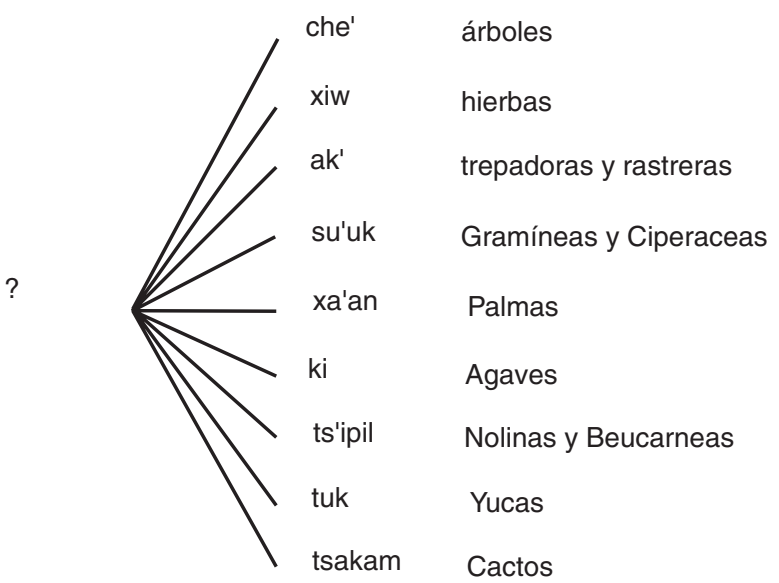

Figura 2. Estructura de la clasificación botánica Maya Yucateca.

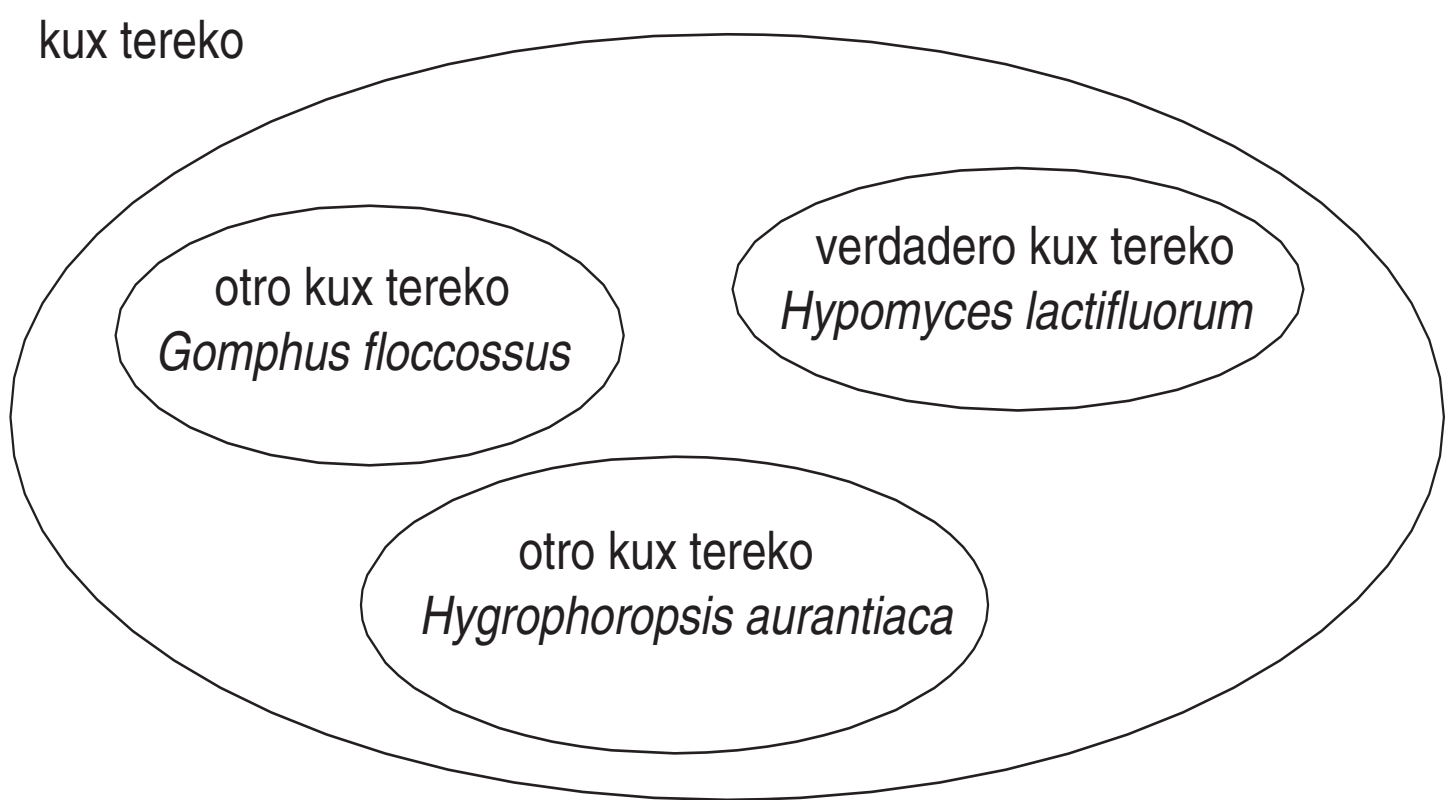

Figura 3. Un ejemplo de clasificación de los géneros hongos en Purhepecha, siguiendo el modelo de clasificación como esferas de influencia sugerido por Bright y Bright (1965) 
cie la que se considera como el verdadero kux tereko, mientras que las otras dos se incluyen en el mismo taxon por que se le parecen o son "compañeras" de ella (Mapes et al. 1981).

Diversos autores han hecho planteamientos alternativos al modelo de clasificación jerárquica de Berlin y colaboradores (1973) los cuales podrían contribuir a explicar las variantes arriba descritas. Así, según Bright y Bright (1965), las taxonomías tradicionales no son en realidad sistemas jerárquicos como la taxonomía Lineana, sino que más bien se pueden concebir como esferas de influencia donde los taxa son agrupados alrededor de una serie de tipos de organismos en función de su parecido. Para Hunn (1976) los seres humanos perciben los organismos como existentes en un espacio clasificatorio y los taxa biológicos podrían ser generados por inducción. Coincidentemente, Randall (1976) ha planteado que el árbol taxonómico no existe realmente en la mente de los individuos y que en realidad los taxa genéricos descritos por Berlin son la base real de un sistema inductivo a partir del cual una persona puede hacer inferencias para clasificar cosas nuevas en un sistema cultural. Un aspecto importante de este debate etnobiológico se refiere a la naturaleza del conocimiento tradicional y las clasificaciones folk. Para una serie de autores quienes siguen un enfoque utilitarista-funcionalista, el conocimiento etnobiológico se origina en la necesidad de satisfacer necesidades biológicas y materiales. Se considera que los criterios funcionales son intrínsecos al ordenamiento taxonómico (Morris (1976, 79, 83), que al menos parte de los sistemas de clasificación reflejan un reconocimiento de utilidad en un sentido amplio y, en general, que el conocimento etnobiológico es adaptativo (Hunn 1982, Posey 1984). En contraste, otros autores sostienen una visión intelectualista del conocimiento etnobiológico. Derivado fundamentalmente del análisis estructuralista desarrollado por Claude Levi-Strauss en su obra "El Pensamiento Salvaje" (Levi-Strauss 1966), el enfoque intelectualista sostiene que el carácter específico del mundo vegetal y animal es la fuente inicial de impulso para las clasificaciones, pero que el propósito de éstas no es uno práctico; el fin es más bien la satisfacción de requerimientos intelectuales. Cercano, pero significativamente diferente en interpretación, el enfoque cognoscitivo desarrollado por Berlin
(1992) sostiene que las sorprendentes similitudes en estructura y contenido entre los sistemas de clasificación biológica de sociedades muy diferentes son la expresión de la capacidad común a todos los seres humanos de apreciar en forma ineludible y mayormente inconsciente la estructura inherente a la realidad biológica de su entorno natural.

La explicación de la estructura de las clasificaciones biológicas tradicionales mesoamericanas en el contexto del debate arriba mencionado rebasa los límites de este trabajo; sin embargo debe señalarse que las incongruencias existentes entre varias clasificaciones biológicas mesoamericanas y los principios generales propuestos por Berlin y colaboradores (1974 y 1992) sugieren la necesidad de revisar dichos principios a la luz de nuevos estudios detallados y comparativos no sólo entre las culturas mesoamericanas sino también entre otras culturas del continente, tales como las de la región andina.

\section{Patrones de Uso de las Plantas}

Estudios realizados por numerosos autores en diferentes regiones de México han documentado la utilización de un gran número de recursos vegetales por las poblaciones indígenas y campesinas tradicionales. Entre algunos de los estudios más importantes están los de Aguilar y colaboradores (1994), Alcorn (1984), Argueta y Cano (1993), Barrera y cols. (1976), Baytelman (1982), Bye (1979a), Caballero y Mapes (1985), Casas, Viveros y Caballero (1994), Kelly y Palerm (1952), Felger y Mosser (1983), Lozoya y Lozoya (1992), Lipp (1971), Martínez (1939 y 1959), Martínez y cols. (1995), Messer (1978), Pennington (1963 y 1969), y Zizumbo y Colunga (1982). La información etnobotánica proporcionada por tales estudios ha sido recopilada y organizada por los autores de este trabajo y por otros colegas del Jardín Botánico de la Universidad Nacional Autónoma de México durante los últimos diez años en una base de datos, la cual es denominada Base de Datos Etnobotánicos de Plantas Mexicanas (BADEPLAM). Esta base de datos reúne información de la literatura, los herbarios y las colectas de campo realizadas por los investigadores del propio Jardín Botánico. Hasta el presente, BADEPLAM incluye más de 16.000 registros de usos para un total de 4.000 
especies pertenecientes a 214 familias. Esto corresponde a más del $50 \%$ del total estimado de especies de plantas útiles de México. BADEPLAM se encuentra aún en una fase de desarrollo y tiene algunos vacíos e inconsistencias en la información. No obstante lo anterior, la revisión de la información incluida hasta el momento permite analizar patrones y tendencias en el uso tradicional de las plantas en México. Con base en esto se pueden discutir algunas posibles implicaciones teóricas para el estudio de la interacción entre las sociedades tradicionales de México y sus entorno vegetal.

Las plantas de México son utilizadas para una gran variedad de propósitos, entre los que se incluyen los usos medicinales, comestibles, colorantes, aromatizantes, maderables, combustibles, materias primas para artesanías, forrajes, adhesivos y otros usos diversos. Como puede observarse casi en cualquier inventario de plantas útiles, los usos más frecuentes de las plantas son como alimento y como medicina (fig. 4). Como es conocido, numerosas especies pueden ser utilizadas para más de un propósito. Aunque la mayoría de las especies registradas en BADEPLAM tiene entre uno y cinco usos, es frecuente encontrar especies que tienen más de diez o quince usos diferentes (fig. 5). Como ha sido señalado por algunos autores (Phillips y Gentry 1993), el uso múltiple de una especie puede ser un indicador de la alta importancia cultural de una planta para las poblaciones humanas. En el caso de las plantas mexicanas hemos estimado que más del $50 \%$ tiene entre dos y cinco usos y alrededor del $25 \%$ tiene más de cinco usos distintos.

La comparación de las especies de plantas útiles de acuerdo a su forma biológica indica que la mayor proporción de las plantas útiles de México corresponde a los árboles y a las plantas herbáceas, aunque también es importante el número de arbustos utilizados (fig. 6). Esta proporción podría ser un reflejo de la frecuencia con que ocurren estas formas biológicas entre las plantas en la naturaleza, aunque el alto número de plantas herbáceas utilizadas podría también estar asociado con el disturbio antropogénico, el cual fomenta el desarrollo de este tipo de plantas, las cuales son frecuentemente utilizadas por su propiedades sobre todo medicinales y comestibles. Algo que pro- bablemente también está asociado con esto, es la alta proporción de especies utiles pertenecientes a las familias Leguminosae y Asteraceae. Como puede verse en la figura 7, el número de especies útiles de estas dos familias es notablemente más alto que el de cualquier otra familia botánica. Esto puede estar asociado con la elevada frecuencia de plantas con alto valor nutricional y la presencia de compuestos secundarios útiles de acción médica entre las especies de ambas familias. Es posible que las poblaciones indígenas de México perciban de algún modo este fenómeno y que tomen ventaja de la alta ocurrencia de factores nutricionales y farmacológicos favorables en las especies de estas dos familias.

\section{Patrones de manejo de los recursos vegetales}

El aprovechamiento de los recursos vegetales involucra plantas bajo diferentes formas de manipulación de las plantas por las poblaciones locales. De acuerdo con Bye (1993), Caballero (1994), Casas et al. (1996, 1997b) y algunos otros autores, se pueden reconocer tres categorías de plantas de acuerdo a la forma de manejo y el grado de manipulación por las poblaciones humanas. Estas categorías son: a) plantas recolectadas, b) plantas bajo manejo incipiente y c) plantas cultivadas. De este modo, muchas especies son recolectadas o cosechadas en la vegetación natural, pero su explotación involucra algún grado de modificación del hábitat, el cual puede afectar a las poblaciones de plantas involucradas, generalmente aumentando su disponibilidad. Existe una amplia gama de formas de manejo incipiente, las cuales intensifican la acción humana sobre las plantas útiles. Este es el caso de la tolerancia de individuos generalmente arborescentes durante la apertura de terrenos a la agricultura y otras actividades productivas. Por otro lado, la promoción de los individuos de diversas especies consiste en acciones dirigidas a aumentar la distribución y la dispersión de propágulos sexuales y vegetativos de las plantas involucradas. Existen también prácticas de protección de plantas útiles, las cuales involucran la remoción de competidores, la exclusión de depredadores y algunas otras formas de cuidado que dan ventaja a las plantas de interés antropogénico sobre sus competidores silvestres. Finalmente, el cultivo involucra la total modificación 


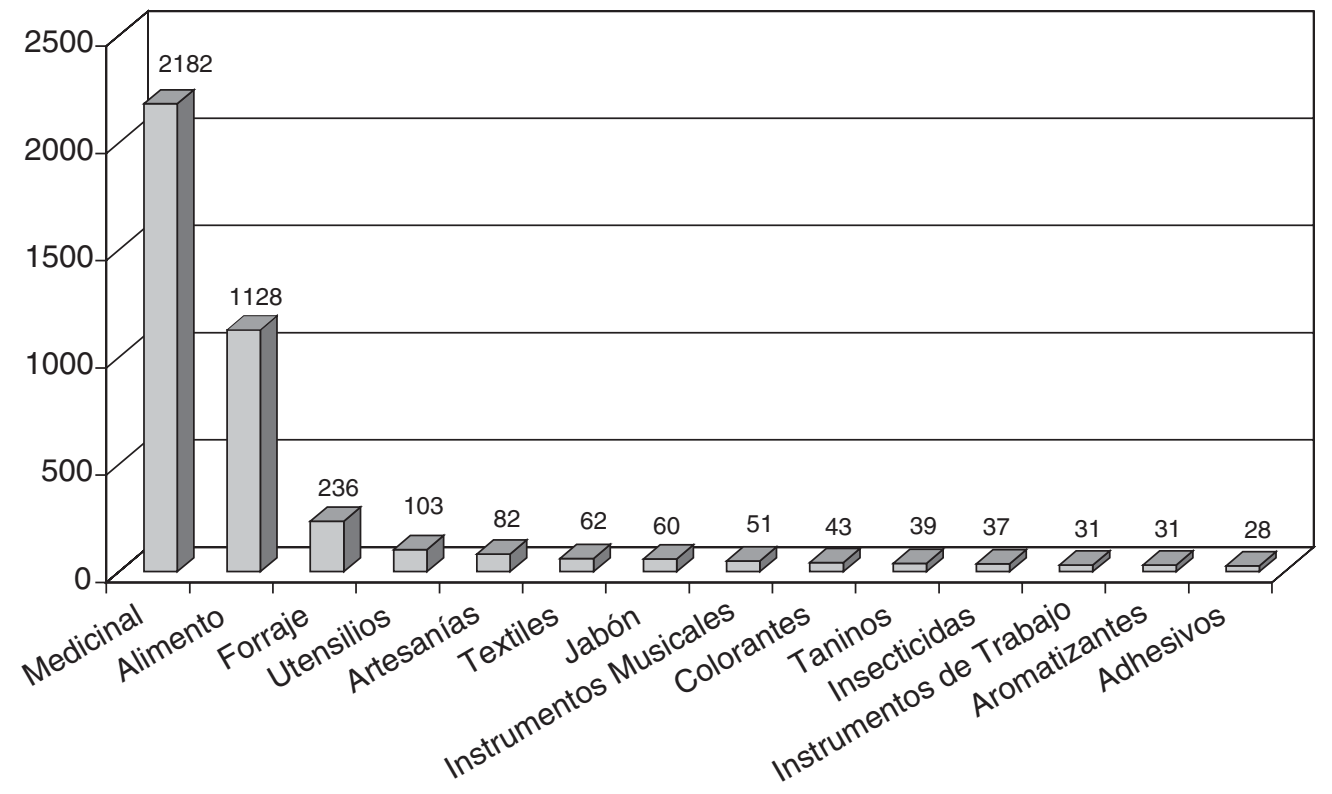

Figura 4. Número de especies de plantas útiles de México, de acuerdo su forma de uso.

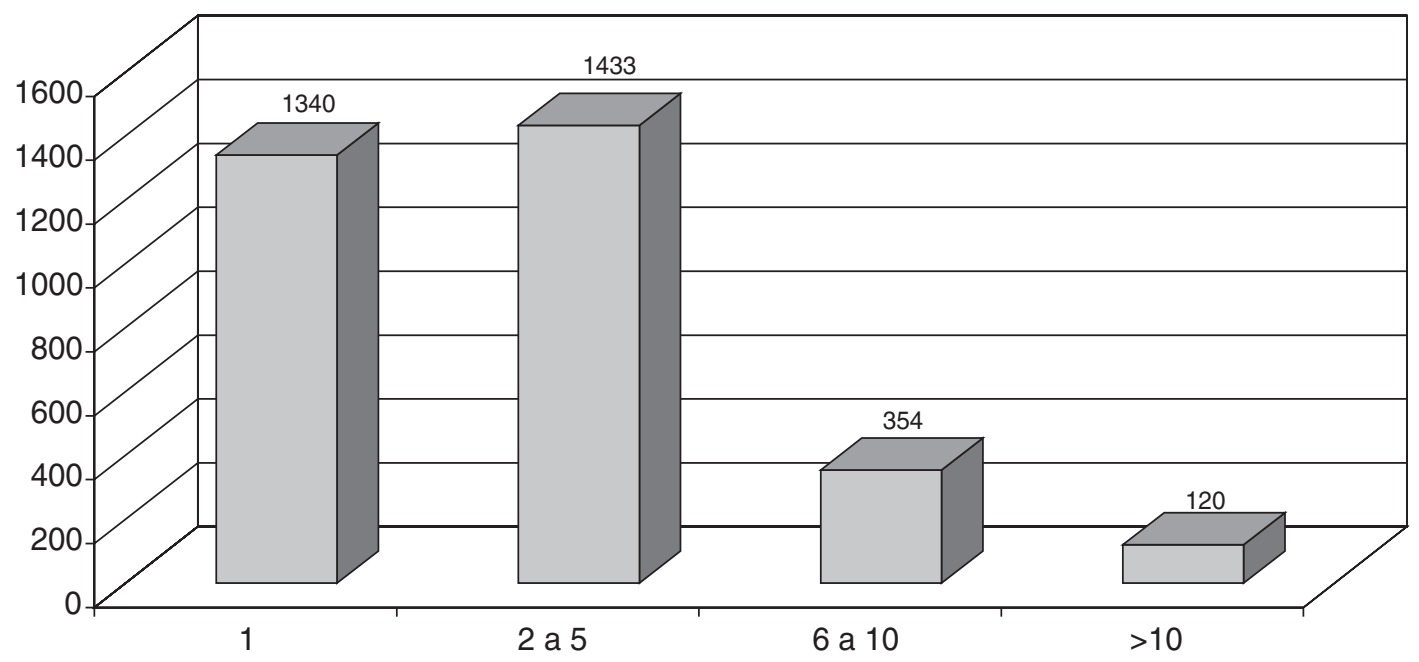

Figura 5. Número de especies de plantas útiles de México de acuerdo al número de usos distintos que tienen. 


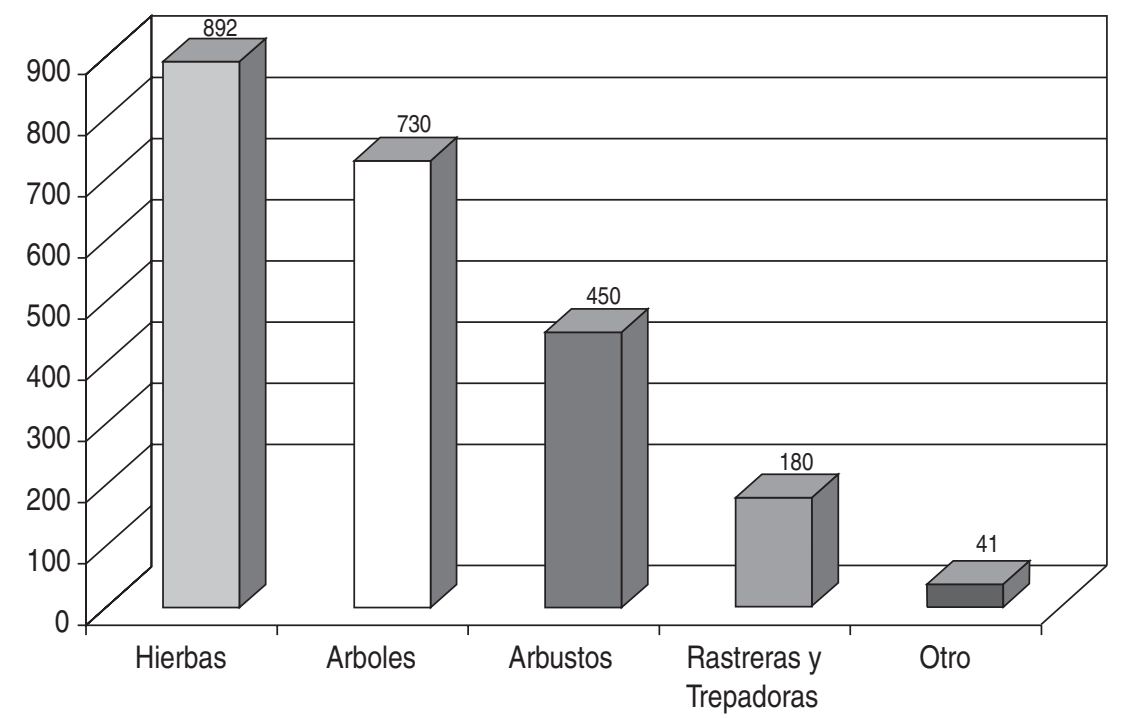

Figura 6. Número de especies de plantas útiles de México de acuerdo a su forma biológica.

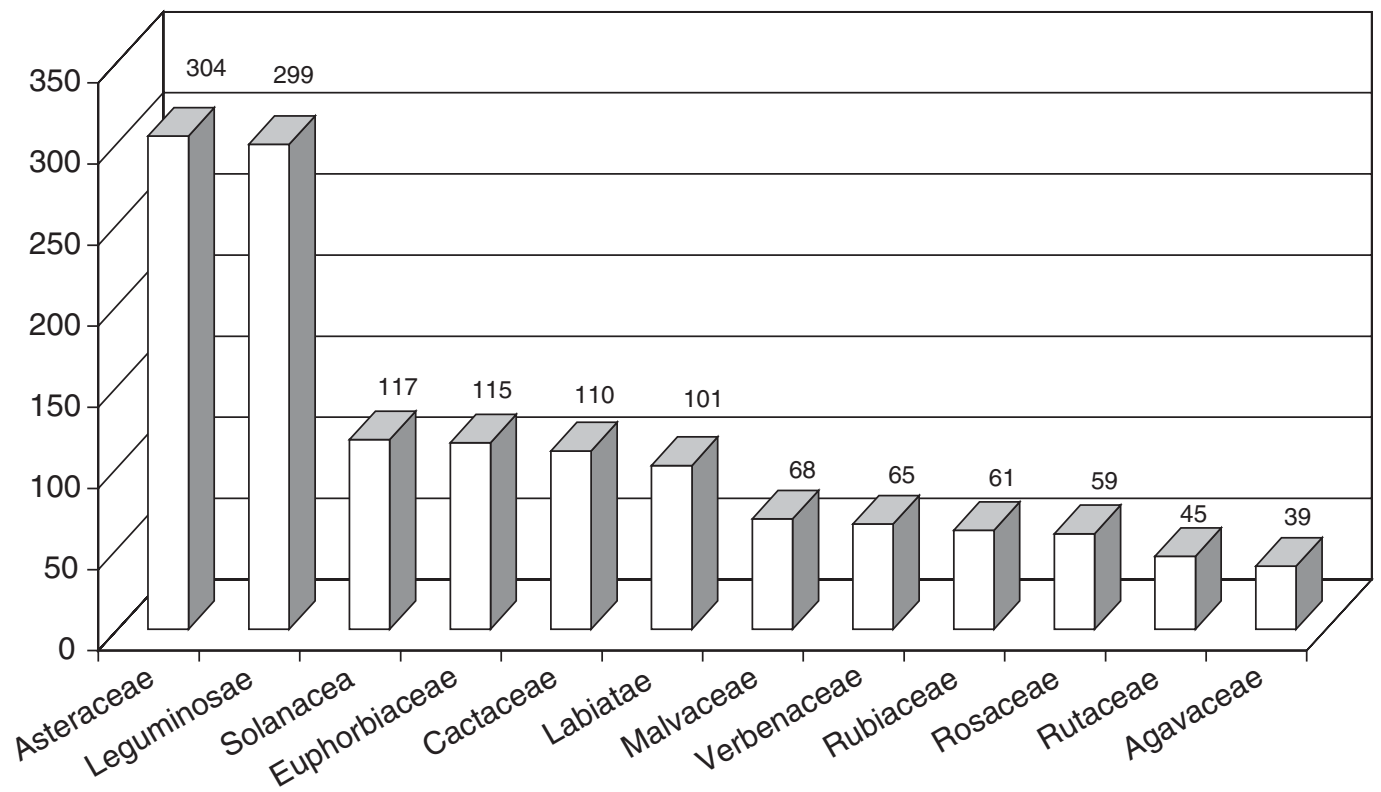

Figura 7. Familias botánicas con mayor número de especies útilizadas en México. 
de las condiciones ambientales con el fin de promover la máxima productividad y reproducción de las plantas involucradas. En relación con esto debe señalarse que es frecuente encontrar especies que están sujetas simultáneamente a varias o, inclusive, a todas las formas de manejo antes mencionadas, ya sea en áreas geográficas diferentes o, dentro de una misma región o localidad.

La información disponible en BADEPLAM permite reconocer patrones de manejo de las plantas útiles. Como puede verse en la figura 8 , la comparación del número de especies utilizadas de acuerdo a su forma de manejo muestra que los recursos utilizados incluyen una gran proporción de plantas silvestres, así como también de plantas bajo alguna forma de manejo incipiente. Diversos estudios de caso han mostrado que el mayor número de especies utilizadas son plantas silvestres. El número de especies bajo alguna forma de manejo incipiente es un poco menor, mientras que las plantas cultivadas constituyen un grupo muy pequeño con respecto a los anteriores. La coexistencia de diferentes formas de manipulación de plantas es particularmente significativa en el caso de las plantas comestibles. Aunque la dieta básica entre las poblaciones rurales incluye sólo un conjunto de especies cultivadas y domesticadas, la alimentación humana se complementa en forma significativa con un gran número de recursos vegetales, la mayoría silvestres y bajo manejo incipiente, los cuales proporcionan vitaminas, minerales y otros importantes nutrimentos. Como puede verse en la figura 9, la proporción de plantas silvestres se incrementa dramáticamente cuando se consideran las plantas medicinales y otros tipos de recursos vegetales; sin embargo, el patrón antes descrito se mantiene prácticamente inalterado. De este modo, el conjunto de recursos vegetales utilizados por las poblaciones tradicionales de México puede verse como una pirámide cuya punta está formada por las plantas cultivadas y domesticadas, mientras que su nivel inferior está formado por un gran número de plantas silvestres, así como plantas bajo manejo incipiente (Caballero 1994).

En conjunto estas formas de manejo de los recursos vegetales han proporcionado la base material de la subsistencia y del desarrollo civilizatorio de las poblaciones indígenas de Mesoamérica. Co- múnmente se asume que el desarrollo de la civilización en Mesoamérica involucró un proceso lineal de evolución y sustitución de un alto número de especies vegetales silvestres por un pequeño conjunto de plantas cultivadas y domesticadas. No obstante lo anterior, el examen de los patrones tradicionales actuales de subsistencia y de utilización de plantas sugiere que la evolución de la civilización involucró la acumulación de conocimiento, tecnologías y recursos vegetales en una estrategia diversificada de subsistencia. Esta estrategia incluye la explotación de recursos vegetales en diferentes niveles de manipulación en un complejo arreglo, el cual es el resultado de un equilibrio existente entre las capacidades tecnológicas de las poblaciones humanas locales y los factores y las limitaciones que impone el medio ambiente.

\section{La manipulación de las plantas y los procesos de domesticación}

Es conocido que Mesoamérica es uno de los principales centros mundiales de origen de la agricultura y de domesticación de plantas. En esta región se domesticaron por lo menos 65 especies de plantas, las cuales además poseen un amplio espectro de variación genética (Caballero 1994). La domesticación de plantas no fue un fenómeno súbito ni tampoco ha concluido. Este es más bien un proceso gradual, continuo y prolongado, en el cual aquellos fenotipos más adecuados para satisfacer las necesidades humanas son seleccionados a lo largo de muchas generaciones. Esta selección antropogénica constituye así un factor que provoca cambios genéticos en las poblaciones vegetales involucradas a lo largo del tiempo en un proceso evolutivo que se inicia con la interacción con las plantas silvestres, continúa con su transformación a plantas arvenses, protegidas, o promovidas, las cuales difieren en cierto grado de las poblaciones silvestres y alcanza sus niveles de mayor intensidad con las plantas domesticadas, las cuales por lo común son morfológica y genéticamente diferentes a sus parientes silvestres (De Wet y Harlan 1965; Schwanitz 1967, Harlan 1976; Bye 1979b; Hawkes 1983). La domesticación de plantas y la manipulación de la variabilidad genética de las plantas es todavía un proceso actual y multidireccional en México (Bye 1981; Casas et al. 1998). Todavía en el presente los agricultores 


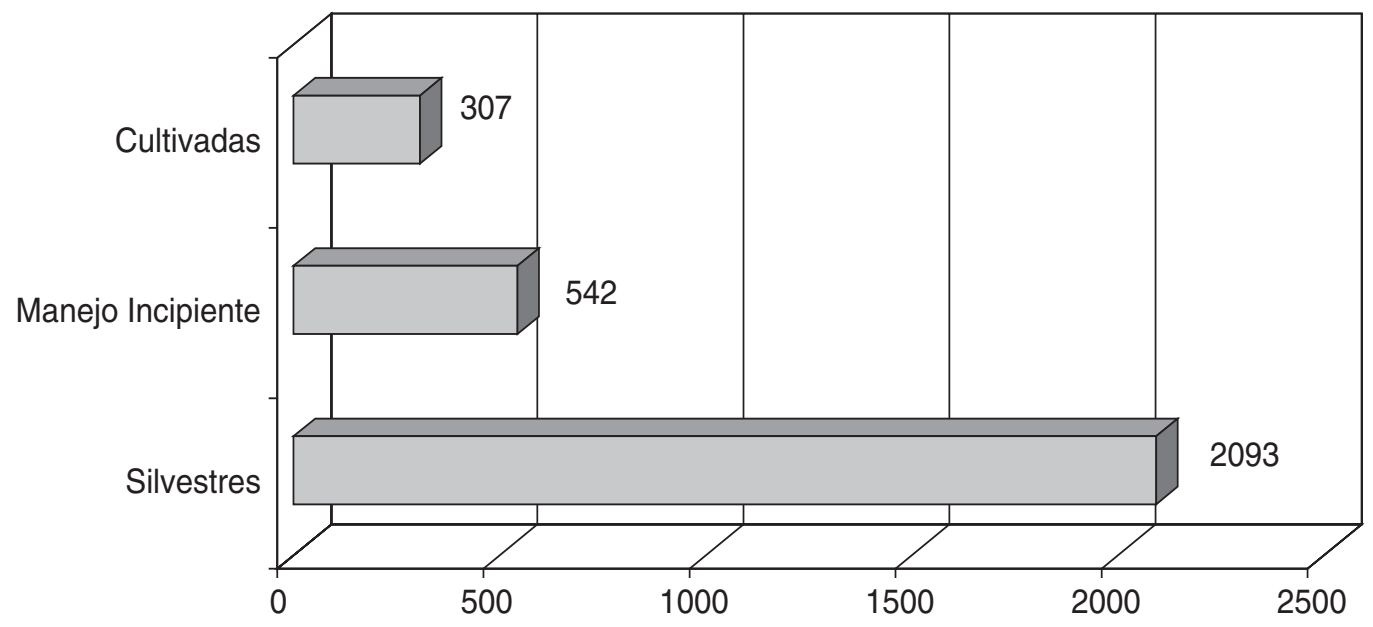

Figura 8. Número de especies de plantas útiles de México de acuerdo a su forma de manejo. No se incluyen especies ornamentales.

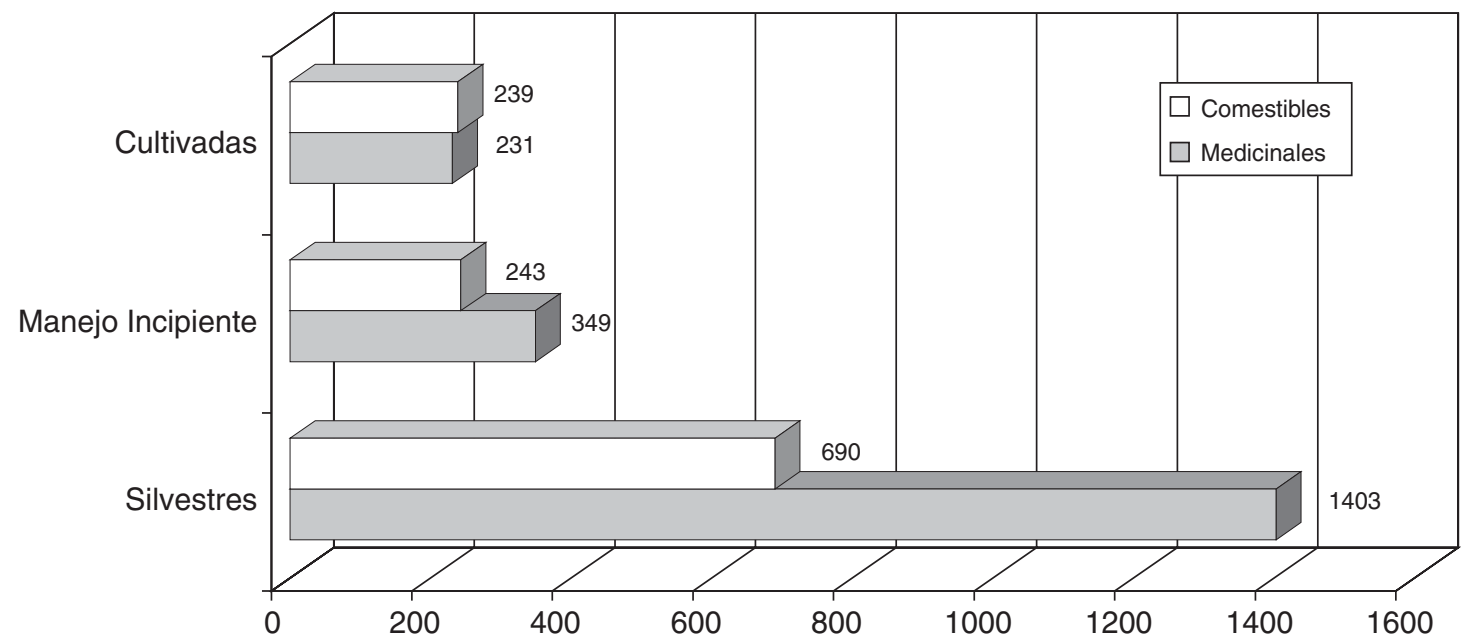

Figura 9. Número de especies de plantas medicinales y comestibles de México de acuerdo a su forma de manejo. 
indígenas continúan la selección de nuevos genotipos de cultivos básicos, como el maíz (Zea mays) y frijol (Phaseolus vulgaris), para adaptarlos a diferentes condiciones climáticas, ecológicas, tecnológicas y económicas (Hernández 1978; Mapes et al. 1996, et al. 1977b).

De acuerdo con investigaciones recientes, las formas de manejo de las plantas descritas arriba, particularmente la tolerancia, protección y promoción de individuos y poblaciones parecen ser también formas actuales de domesticación, las cuales no implican necesariamente el cultivo de las plantas involucradas. Esto es particularmente cierto en el caso de un importante número de plantas perennes, como en el caso del guaje colorado (Leucaena esculenta subsp. esculenta), una leguminosa cuyas semillas son ampliamente consumidas como alimento en el centro de México (Casas y Caballero 1996). Los Mixtecos de la región de la Montaña de Guerrero cosechan las vainas de guaje colorado, tanto de los árboles silvestres como de aquellos árboles que son dejados en pie cuando se abre un terreno para la agricultura o el pastoreo.
La práctica de dejar individuos de guaje en pie es selectiva, ya que sólo aquellos árboles que se consideran mejores son dejados en pie, mientras que los demás son eliminados. Al comparar la morfología de las vainas y las semillas de muestras de individuos de acuerdo a su forma de manejo, dichos autores encontraron diferencias estadísticamente significativas en aquellos caracteres relacionados con su utilización por la población local (Tabla 1). De este modo Casas y Caballero (1996) encontraron que, si bien ambos tipos de árboles presentan un número similar de semillas por vaina, tanto las vainas como las semillas de los individuos tolerados eran significativamente más grandes. En contraste, las semillas de los individuos tolerados presentaron un mayor procentaje de predación por brúquidos. Esto sugiere que mediante un proceso de selección in situ basado en una estrategia de pérdidas y ganancias, los campesinos Mixtecos están aumentando la frecuencia y promoviendo aquellos fenotipos con características más deseables, aunque esto signifique que dichos fenotipos tengan también algunas características no deseadas.

Tabla 1. Comparación de los valores promedio de algunas características de una muestra de vainas y semillas de Leucaena esculenta subsp. esculenta, de acuerdo a su forma de manejo por los Mixtecos de la Montaña de Guerrero, México. Para cada forma de manejo casi se muestrearon 200 vainas de un total de 20 individuos. Basado en Casas y Caballero (1996) y Casas y colaboradores (1998).

\begin{tabular}{lccl}
\hline & \multicolumn{2}{c}{ Forma de manejo } & Nivel de \\
\cline { 2 - 3 } & Tolerados & Silvestres & significancia \\
\hline Tamaño de vaina (cm2) & 21.5 & 27.7 & 0.02 \\
Tamaño de semilla (cm2) & 0.48 & 0.66 & 0.0001 \\
Número de lóculos por vaina & 12.4 & 12.2 & 0.6 \\
Número de semillas & 14.9 & 14.5 & 0.5 \\
Número de semillas depredadas & 3.9 & 6.7 & 0.008 \\
\hline
\end{tabular}


Una situación similar ha sido descrita por Casas y colaboradores $(1998,99)$ en el caso del xoconostle (Stenocereus stellatus), una cactacea columnar de las zonas áridas del centro de México, la cual proporciona frutos comestibles muy apreciados por la población local. Aunque no han sido suficientemente documentados, estos patrones de variación morfológica asociados a las forma de manejo en plantas perennes pueden observarse también en numerosas especies de plantas perennes en Mesoamérica, tales como el guamuchil (Pithecellobium dulce), el nance (Byrsonima crassifolia) y el ciruelo (Spondias mombin y S. purpurea). Lo anterior, sugiere la existencia en Mesoamérica de procesos actuales de domesticación in situ, los cuales no necesariamente implican el cultivo de las plantas como una vía para la selección de los fenotipos deseados, sino que la selección antropogénica se realiza en el hábitat original de las plantas, mediante el manejo de sus poblaciones. Estas poblaciones, además, no son manejadas en forma aislada, sino como parte de elaborados sistemas agrosilvopastoriles, los cuales dan lugar a un complejo paisaje antropogénico donde prácticamente todos sus elementos tienen algún papel en la economía local y son, en alguna medida, el resultado de un prolongado y cuidadoso proceso de selección y manipulación por los seres humanos. Con base en lo anterior, puede decirse las prácticas tradicionales de manejo de los recursos vegetales en
Mesoamérica, si bien a menudo conllevan la eliminación de porciones significativas de los ecosistemas naturales, han generado -y lo siguen haciendo- una importante cantidad de diversidad biológica a la ya existente por causas naturales.

\section{Consideraciones finales}

La comparación de las formas de conocimiento, clasificación y manejo de las plantas en México, y en general en Mesoamérica, con las de otras regiones de América y del mundo tiene una gran relevancia teórica en Etnobiología. La interacción entre los seres humanos y su entorno biológico es sin duda un proceso moldeado por una compleja matriz de factores biológicos, sociales y culturales, los cuales determinan que dicha interacción presente características particulares, tanto en el espacio como en el tiempo. El ejemplo de México, sin embargo, sugiere la posible existencia de patrones comunes en la interacción entre las sociedades tradicionales en general y su entorno ecológico. Desde esta perspectiva, el estudio comparativo de las formas de conocimiento, utilización y manejo de los recursos biológicos en diferentes contextos culturales, ecológicos y geográficos puede contribuir a entender los principios biológicos y socioculturales que subyacen la interacción entre los seres humanos y su medio ambiente. 


\section{BIBLIOGRAFIA}

ALCORN, J.B. Huastec Mayan Ethnobotany. 1984 University of Texas Pressm Austin Press. págs 23-39.

AGUILAR, A., J.R. CAMACHO, S. CHINO y P. JACQUEZ 1994 \& M.E. LÓPEZ. Herbario medicinal del IMSS: Información etnobotánica. México, Instituto Mexicano del Seguro Social.

ARGUETA, A. Etnozoología purhé. historia, utiliza1988 ción y nomenclatura Purhepecha de los animales. Tesis. Biólogo, Facultad de Ciencias, UNAM. México.

ARGUETA, A. y L. CANO. El atlas de las plantas de la medi1993 cina tradicional mexicana. La Investigación científica de la herbolaria medicinal mexicana. México, Instituto Nacional Indigenista 3.

BARRERA, A. La taxonomía botánica Maya. Anales 1979 de la Sociedad mexicana de Historia de la Ciencia y la Tecnología 5:21-34.

BARRERA, A., A. BARRERA-VAZQUEZ y R.M. LOPEZ1976 FRANCO. Nomenclatura etnobotánica maya. México, INAH-SEP.

BAYTELMAN, B. Etnobotánica del Estado de Morelos. 1982 México, SEP-INAH.

BERLIN, B. Ethnobiological classification.Princi1992 ples of categorization of plants and animals in traditional societies. Princeton, New Jersey: Princeton University Press, 335p.

BERLIN, A.E. y B. BERLIN. Medical ethnobiology of the 1996 highland Maya of Chiapas, México The gastrointestinal diseases. Princeton University Press, New Jersey.

BERLIN, B. D.E. BREEDLOVE y P.H. RAVEN. General 1973 principles of classification and nomenclature in folk biology. American Anthropologist 75: 214-242.

BERLIN, B. D.E. BREEDLOVE y P.H. RAVEN. Principles 1974 of Tzeltal plant classification. Academic Press. N.Y.

BRIGHT, J.O. y W. BRIGHT. Semantic structures in North1965 western California and the Sapir-Worf hypothesis.American Anthropologist 67: $249-258$
BYE, R

$$
\text { 1979a }
$$

BYE, R.

BYE, R. 1981

Quelites -ethnoecology of edible greens- Past, present and future. Journal of Ethnobiology 1: 109-123.

BYE, R. tion of plants in Mexico. In T.P. Ramamoorth, R. Bye, A. Lot \& J. Fa (eds.). Biological diversity in Mexico. Oxford Univ. Press, NY.

CABALLERO, J. La dimension culturelle de la diversite 1994 vegetale au Mexique. Journal d' Agriculture. Traditionelle et de Botanique. Appliquee, nouvelle série, Vol. XXXVI (2): 1-12.

CABALLERO, J. y C: MAPES. Gathering and subsistence patterns among the Purhepecha Indians of Mexico, Journal of Ethnobiology 5(1): $31-47$

CASAS, A., J.L. VIVEROS y J. CABALLERO. Etnobotánica 1994 mixteca: sociedad, cultura y recursos naturales en la Montaña de Guerrero. México: CONACULTA e Instituto Nacional Indigenista.

CASAS, A. y J. CABALLERO. Traditional management and 1996 morphological variation in Leucaena esculenta (Moc. et Sessé ex A.DC.) Benth. (Leguminosae: Mimosoidae) in the Mixtec Region of Guerrero, México. Economic Botany 50 (2): 167-181.

CASAS, A., C. VAZQUEZ, J.L. VIVEROS y J. CABALLE1996 RO. Plant management among the Nahua and the Mixtec in the Balsas River basin, Mexico: an ethnobotanical approach to the study of domestication. Human Ecology 24 (4): 455-478.

CASAS, A., B. PICKERSGILL, J. CABALLERO, y A. VA1997a LIENTE-BANUET. Ethnobotany and domestication of the "xoconochtli" Stenocereus stellatus (Cactaceae) in the Tehuacán Valley and la Mixteca Baja, México. Economic Botany 51(3):279292. 
CASAS, A., J. CABALLERO, C. MAPES y S. ZARATE. 1997b Manejo de la vegetación, domesticación de plantas y origen de la agricultura en Mesoamérica. Boletín de la Sociedad Botánica de México 61: 31-47

CASAS, A., A. VALIENTE-BANUET y J. CABALLERO. 1998 La domesticación de Stenocereus stellatus (Pfeiffer) Riccobono (Cactaceae). Boletín de la Sociedad Botánica de México 62: 129-140

CASAS, A., J. CABALLERO, A. VALIENTE-BANUET, J.A. 1999 SORIANO y P. DAVILA. Morphological variation and the process of domestication of Stenocereus stellatus (Cactaceae) in central Mexico. American Journal of Botany 86 (4): 522-533.

DE WET, J.M.J. y J.R. HARLAN. Weeds and domesticates: 1975 evolution in the man-made habitat. Economic Botany 29: 99-107.

FELGER, R. y M. MOSSER. People of the dessert and the 1983 sea. Ethnobotany of the Seri Indians. University of Arizona Press, Tucson.

FRAKE, C.O. The ethnographic study of cognitive 1962 systems. En: Gladwin, T. \& W.C. Sturtevant (eds.) Anthropology and human behavior 72-93.Anthropological Society of Washington, Washington.

HARLAN J. R. Crops and Man. American Society of 1975 Agronomy. Madison

HAWKES, J.G. The diversity of crop plants. Harvard 1983 University Press. Cambridge.

HERNANDEZ, X. E. Biología agrícola. C.E.C.S.A. México. 1985

HUNN, E. 1976

Toward a perceptual model of folk biological classification. American Ethnologist 3: 508-524.

HUNN, E. 1977

Tzeltal Folk Zoology: The classification of discontinuities in nature. New York: Academic Press, N.Y.

HUNN, E.

The utilitarian factor in folk biological 1982 classification. American Anthropologist 84: 830-847:

KELLY, I. y A: PALERM. The Tajin Totonac. Smithsonian 1952 Institution. Institute of Social Anthropology. Publication $n^{\circ} 13$, Washington.

LEVY-STRAUSS, C. El pensamiento salvaje. Fondo de Cul1972 tura Económica, México.
LOZOYA, L. X. y M. LOZOYA. Flora medicinal de Méxi1992 co. Primera parte: plantas indígenas. Instituto Mexicano del Seguro Social. México.

LIPP, E. Oaxaca, México. Economic Botany 25: 234-244 (25): 234-244.

MARTINEZ-ALFARO, M. A. et al. Catálogo de plantas úti1995 les de la Sierra Norte de Puebla. México, Instituto de Biología, Universidad Nacional Autónoma de México.

MARTINEZ, M. Las plantas medicinales de México. 1939 México, Ediciones Botas.

MARTINEZ, M. Plantas útiles de la flora mexicana. 1959 México, Ediciones Botas.

MAPES C., G. GUZMAN y J. CABALLERO.Etnomicologia 1981 Purhepecha: El conocimiento y uso de los hongos en la Cuenca de Pátzcuaro. México, D.F.: Dirección General de Culturas Populares, S.E.P., Serie Etnociencia $\mathrm{N}^{\circ} 2$.

MAPES C., G. GUZMAN y J. CABALLERO. Elements of 1982 Tarascan mycological classification. Journal of Ethnobiology 1 (2): 231-237.

MAPES, C., J. CABALLERO, E. ESPITIA, y R.A. BYE.

1996 Morphophysiological variation in mexican species of amaranthus: Evolutionary tendencies under domestication. Genetic Resources and Crop Evolution 43 (3): 283-290.

MESSER, E. Zapotec plant knowledge: classifica1978 tion, uses, and communication about plants in Mitla, Oaxaca, Mexico. Memoirs of the Museum of Anthropology University of Michigan, 1978. $\mathrm{N}^{\circ} 10$ parte 2, Ann Arbor.

MORRIS, B. Whither the savage mind? Notes on the 1976 natural taxonomies of hunting and gathering people. Man 11: 542-557.

MORRIS, B. $\quad$ Symbolism and ideology: Thoughts 1979 around navajo taxonomy and symbolism En Ellen, D.R. \& D. Reason (eds.). Classifications in their social context. 117-138. Academic Press, London.

MORRIS, B. The pragmatics of folk classification. 1983 Journal of Ethnobiology 4: 45-60.

PENnington, C. The Tarahumara of Mexico. The en1963 vironment and material culture. The University of Utah Press, Salt Lake City. USA. 
PENnington, C. The Tepehuan of Chihuahua. Their 1969 material culture. The University of Utah Press, Salt Lake City.

PHILLIPS, O.L. y A.H. GENTRY. The useful plants of Tam1993 bopata, Peru I.Statistical hypothesis tests with a new quantitative technique. Economic Botany 47: 33-43.

POSEY, D.A. Hierarchy and utility in a folk botanical 1984 taxonomic system: Patterns in the classification of Arthropods by the Kayapó Indians of Brazil. Journal of Ethnobiology 4: 123-134.

RZEDOWSKI, J. Vegetación de México. Trillas. Méxi1978 co.

RZEDOWSKI, J. Diversity and origins of the phaneroga1993 mic flora of Mexico. En: Ramamoorthy T.P, R. Bye, A. Lot \& J. Fa (eds.) Biological Diversity of México. Oxford University Press, N.Y., 129-144.
TOLEDO, V.M., J. CABALLERO, C. MAPES, N. BARRE1980 RA, A. ARGUETA y M.A. NUÑEZ. Los Purhepechas de la Cuenca del Lago de Pátzcuaro: Una Aproximación Ecológica. América Indígena 40 (1): 17-55.

TOLEDO, V.M. La diversidad biológica de México. Cien1988 cia y Desarrollo 81: 17-30

RANDALL, R. How tall is a taxonomic tree? Some 1976 evidence for dwarfism. American Ethnologist 3: 543-553.

WILLIAMS, D. E. A review of sources for the study of $1990 \quad$ Nahuatl plant classification. Advances in Economic Botany (8): 249-270.

ZIZUMBO, D. y P. COLUNGA. Los Huaves: La apropia1982 ción de los recursos naturales. México, Universidad Autónoma de Chapingo. 